\title{
THE LAST GENERATION OF ELECTRONIC STETHOSCOPES: THE INTERNET TELE-STETHOSCOPE
}

\section{GIORGIO A.}

Politecnico di Bari, Dipartimento di Elettrotecnica ed Elettronica, via E. Orabona, 4, 70125 Bari, Italy. ${ }^{*}$ Corresponding Author: Email- a.giorgio@poliba.it

Received: November, 12, 2011; Accepted: March 15, 2012

\begin{abstract}
This paper deals with the problem of objectification in cardio-pulmonary auscultation, simple, not-invasive and essential diagnostic test, but currently not objectifiable, and the ability to perform real-time teleauscultation in order to improve the diagnostic potential of telemedicine, at present mainly limited to the teleelectrocardiography, the teleconsultation and the sending of delayed reports. For this purpose, a medical device, named Internet Tele-Stethoscope, consisting of hardware and software, has been designed and successfully validated .

Key words- Electronic stethoscope, chest auscultation, lung sounds, heart sounds, teleauscultation, teleconsultation, telemedicine, home care
\end{abstract}

Citation: Giorgio A. (2012) The Last Generation of Electronic Stethoscopes: The Internet Tele-Stethoscope. International Journal of Bioinformatics Research, ISSN: 0975-3087 \& E-ISSN: 0975-9115, Volume 4, Issue 1, pp.-249-253.

Copyright: Copyright@2012 Giorgio A. This is an open-access article distributed under the terms of the Creative Commons Attribution License, which permits unrestricted use, distribution, and reproduction in any medium, provided the original author and source are credited.

\section{Introduction}

Auscultation of lung sounds and heart sounds is one of the classic diagnostic methods commonly used in medical practice, and runs through stethoscope.

Although useful, non-invasive and of rapid implementation, it is a diagnostic test particularly sensitive to the physician's subjectivity, both in the reception quality of biological sounds and in their interpretation for the purpose of diagnosis.

From several years by now amplified electronic stethoscopes with noise filtering systems have been developed to improve diagnostic accuracy.

As far as these solutions have allowed a better perception of biological sounds by the physician and even the recording and the archiving of sounds acquired, however there remains the problem of subjectivity by which the interpretation of sounds is conditioned, and the need for the doctor to be close to the patient, since it is currently impossible the reliable real-time remote transmission of sounds, but only deferred, or upon registration.

With the designed device, we meet these requirements obtaining an objective auscultatory diagnosis in real-time, even by a remotely located physician (tele-auscultation), by using real-time spectral analysis of the biological sounds. In fact there is a deterministic relationship between the spectrum of biological sounds and pathologies [1]-[2].

The device, which represents a technological evolution in comparison to electronic stethoscopes, allows you to transmit sounds at any distance without suffering any distortion or alteration which diminishes the accuracy of diagnosis and to correlate, in real-time, spectral analysis of heart and lung sounds with their diseases, thereby allowing an objective diagnosis.

The device can also store the recorded sounds useful for comparisons over time to monitor the evolution of diseases.

The device was successfully validated for both heart sounds and lung sounds and showed a considerable educational value for physicians in training who need to gain experience in correlating correctly and objectively biological sounds to diseases.

In this paper, after an overview of the cardio-pulmonary sounds and their spectral properties (sections 2 and 3), which allow their objectification, we describe the device design (section 4) and the results obtained (section 5 ), then the conclusions and directions for future development are given. 


\section{Origin and spectral properties of respiratory sounds}

Organic sounds originate from mechanical vibrations in compressible media and are transmitted through tissues as sound waves. The lung sounds, in particular, are generated in large airways, where high speed and air turbulences induce vibrations along the walls of the breathing tubes. Such vibrations are then transmitted through the tissue of the lungs and the chest walls, up to the surface, where they can easily be perceived with the aid of a stethoscope. The generation of lung sounds is directly related to the speed of airflow and to the architecture of the airways. The velocity of air flow is mainly determined by pulmonary ventilation and cross-section of the airways at every level of the lungs.

Terminal airways or alveoli illnesses are responsible for changes in the lung sounds heard on the surface, because the diseased tissues are responsible for the increase or decrease of the sound transmitted. Differences in intensity and characteristics of perceived sounds are, therefore, of great help in identifying specific diseases of the chest, as changes in tissue density involve acoustic attenuation, reflection and refraction of sound waves. Furthermore, the properties of the sound heard on the surface are determined by factors that affect the sound generation and characteristics of the intermediate tissues: every type of tissue is able to attenuate the sound vibrations of different frequencies and in different quantities, which translates in an alteration of the sound spectrum and a lower amplitude sound in certain frequencies [3-4]. When the acoustic properties of tissues through which the sound propagates differ greatly, as between the air-filled lungs and muscles of the chest wall, much of the sound wave is reflected and sound intensity decreases. For this reason, the large-spectrum sound, generated in the large airways, it is first filtered by the lung parenchyma (the organic material that constitutes the lungs) and chest wall, then reaches into the skin without the high-frequency spectral components: ultimately the lung-thorax system behaves as low-pass filter for respiratory sounds.

When, due to disease, the spongy and full of air tissue of the lungs is replaced by clusters and liquid and becomes a solid mass, the lung-thorax system is capable of transmitting high tones. Therefore, the presence of high frequency components in lung sounds is a symptom of respiratory diseases. The frequency range of the lung sounds in healthy people extends up to $1000 \mathrm{~Hz}$, although the greatest concentration of power is observed between $60 \mathrm{~Hz}$ and $600 \mathrm{~Hz}$. With increasing age there is a small increase in power in the band between $330 \mathrm{~Hz}$ and $600 \mathrm{~Hz}$. Spectral differences exist between women and men in general, women have tones of breath sounds higher than men.

The best frequency band to obtain the average power is the band between $100 \mathrm{~Hz}$ and $600 \mathrm{~Hz}$, because here we have a probability of error less than the band between $100 \mathrm{~Hz}$ and $800 \mathrm{~Hz}$, and the information that is lost is negligible because the power is almost entirely concentrated in the first band.

In addition to the chest, another common position to auscultate breath sounds with a stethoscope is on the neck in the hollow above the breastbone to the extrathoracic trachea. The sounds recorded here are called tracheal sounds. These sounds have an amplitude large enough, on average, greater than about $20 \mathrm{~dB}$ at low frequencies, and have a frequency range wider than the sounds listened from the chest. The intensity of breath sounds is greater during expiration than during inspiration. They do not show a peak at low frequency, rather they extend nearly flat in amplitude between $100 \mathrm{~Hz}$ and $700 \mathrm{~Hz}$, where the first peak appear, then decrease down to the second peak at about $1500 \mathrm{~Hz}$.

However, the spectral characteristic of tracheal sounds varies considerably among patients, and also depends on the width of the trachea and physiology and physique of the patient. It has been shown that patients with tracheal stenosis have a spectrum with a significant increase both in power, with a peak at about 1 $\mathrm{KHz}$, and in bandwidth, with spectral power that extends from 600 $\mathrm{Hz}$ to $1300 \mathrm{~Hz}$.

\section{Origin and spectral properties of heart sounds}

Heart sounds are produced by the movement of the valves and the turbulent flow of blood.

The normal heart sounds consist of two parts: a first pulse due to the closure of the atrio-ventricular valves, which denotes the complete passage of blood in the ventricle, a second pulse due to the closing of the valves between ventricles and great vessels, which denotes the complete passage of blood from the ventricles to the aorta and pulmonary arteries.

The blood is pushed through the arteries by the mechanical movement of the heart, but if the arteries are partially occluded blood flow is disturbed, and this creates turbulence in the arterial. Following this pressure variations are produced and heart sounds called murmurs due to blockage of his arteries, can be heard on the surface of the chest, matching the outbreaks of auscultation. The murmurs are high frequency sounds that cover a range between $250 \mathrm{~Hz}$ and $1000 \mathrm{~Hz}$. They become appreciable when the occluded area of the arteries reaches $75 \%$ of the total. Several studies confirm that the frequency range between 400 and $800 \mathrm{~Hz}$ is associated with coronary artery stenosis.

The spectrum of non-pathological heart frequencies extends between 10 and $400 \mathrm{~Hz}$, but the range that provides more info is between 20 and $150 \mathrm{~Hz}$. The lung sounds and the muscle noise contained within the bandwidth of $100 \mathrm{~Hz}$ interfere with the heart sounds and this makes difficult the distinction necessary for diagnosis. With the use of electronic devices and appropriate numerical algorithms [5-6] it is possible to distinguish the heart sounds and lung sounds by filtering out noise.

\section{Description of the designed device}

The designed device is a more advanced version of a standard electronic stethoscope and permits: the acquisition of lung sounds and heart sounds as described in section 3 , the spectral analysis in real time and real-time transmission via personal computer (PC) to a remote $\mathrm{PC}$, both for sounds and for the spectrum. The device, schematically depicted in Figure 1, is PC-based and consists of a hardware component consisting of a sensor and a filter-amplifier (similar to a standard electronic stethoscope) and a software interface.

The hardware side, respiratory and cardiac sounds are picked up by the microphone capsule, housed in a bell stethoscope to be affixed to the patient's chest. The transduced signal is amplified and filtered. The user can select the type of filtering to be applied to the signal, so as to enhance the cardiac and respiratory components. The conditioned signal is then sampled and quantized by a form of $A / D$ conversion. 


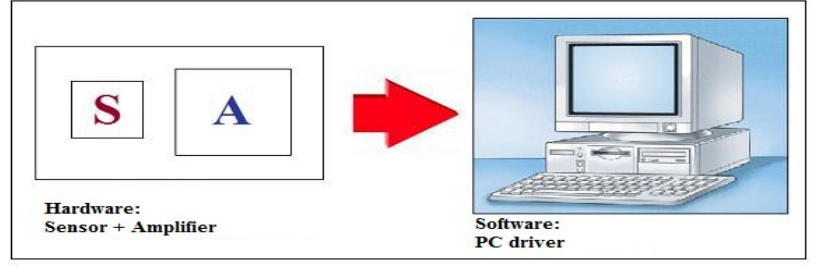

Fig. 1- Drawing of the designed device

The system is powered by a rechargeable battery in the device itself using a special charger.

It has not been allowed the use of any compression algorithm, so as to preserve the quality of the transmitted signal.

The connection to a PC is possible as with a cable to the sound card of the PC as via Bluetooth. A microcontroller is necessary to oversee the management of the device functioning.

The prototype of the internet tele-stethoscope has the same chassis of the typical well known electronic stethoscope but it can also be redesigned assuming the appearance similar to that of a normal PC mouse, as shown in figure 2.

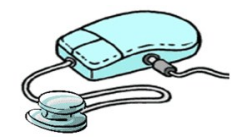

Fig. 2- Possible new mechanical design of the internet telestethoscope, alternatively to the classical chassis

The software allows the acquisition of sound through a PC and real-time transmission of audio streams, via web, to another PC; the software implements, moreover, all the digital filtering necessary to optimize the auscultation including the osculation, the Fourier transform to perform spectral analysis, environmental and muscle noise filtering, heart sounds from lung sounds filtering [78]. The software user interface, shown in figure 3, allows archiving of sounds, playback, deferred analysis, spectrum printing, zoom, sending audio files.

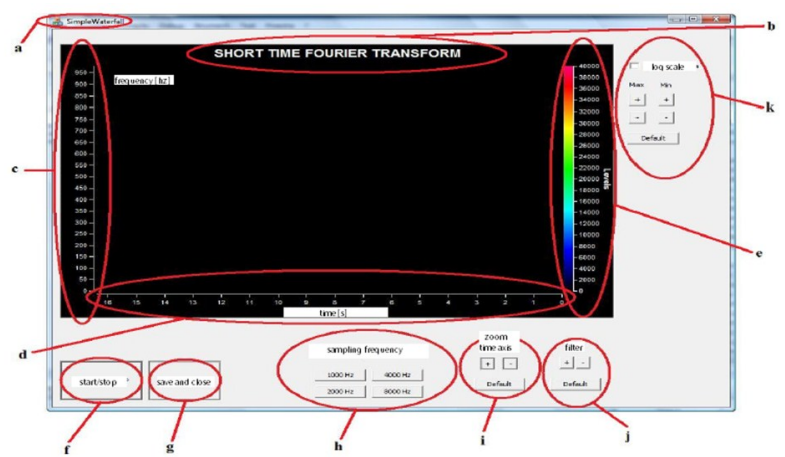

Fig. 3- Software User Interface: (a) Program name. (b) Title. (c) Frequency axis. (d) Time axis. (e) Intensity scale. (f) Start button: to start the program, pause it and restart it. (g) Quit button. (h) Sampling rate selection buttons: 1 - 2 - 4 to $8 \mathrm{kHz}$. (i) Zoom of the times. (j) Filter: allows you to increase or decrease the application of the video filter; if the limit is decreased, the filter is not applied. (k) Management of the intensity scale: allows you to switch between linear and logarithmic scale, and vice versa, and to vary the range of the scale. Move your mouse over the image appear zoom options, files, etc...
Figure 4 shows the principle of real-time transmission and the setup made to validate the device.

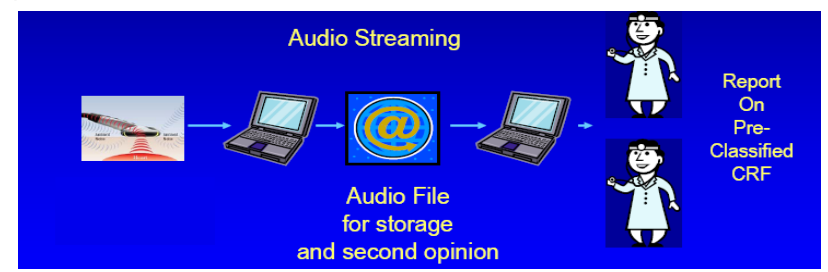

Fig 4- Real time transmission and set-up for validation

The software side, developed in Visual $\mathrm{C}++2008$, allows the acquisition of sound through a PC and real-time transmission of audio streams, via web, to another PC; implements, moreover, all the filtering necessary to optimize the auscultation including the osculation, the Fourier transform to perform spectral analysis, environmental and muscle noise filtering, heart sounds from lung sounds filtering [5-9].

\section{Experimental results}

Several acquisitions of lung sounds and heart sounds have been made, both with the new device and with an electronic stethoscope reference of the best commercially available and compared [10]. The comparable results indicate the reliability of the new diagnostic device.

A few pictures are shown as examples of the sound spectrum. Figure 5 shows the sound spectrum of a tracheal sound acquired and recorded by the reference stethoscope and in Figure 6, the same sound acquired by the new device.

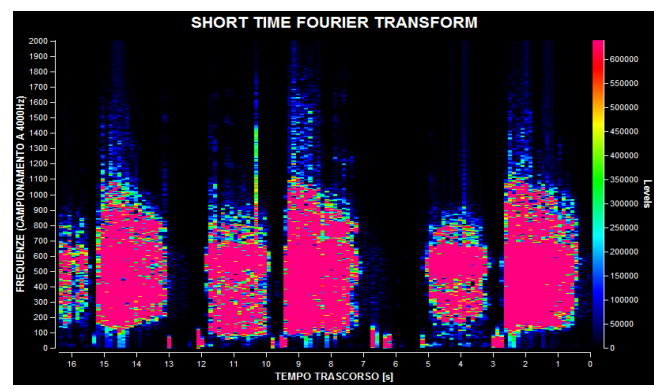

Fig. 5- Sound spectrum of a tracheal sound acquired by the reference electronic stethoscope

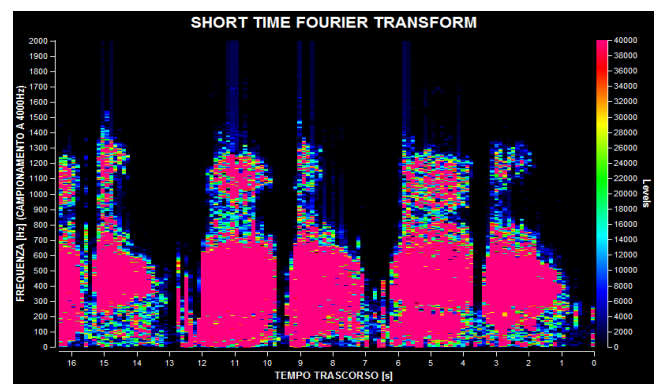

Fig. 6- Sound spectrum of a tracheal sound acquired by the new device

Figure 7 shows the sound spectrum of a heart sound concerning the aortic acquired using the reference electronic stethoscope and in Figure 8, the same sound was acquired by the new device. 


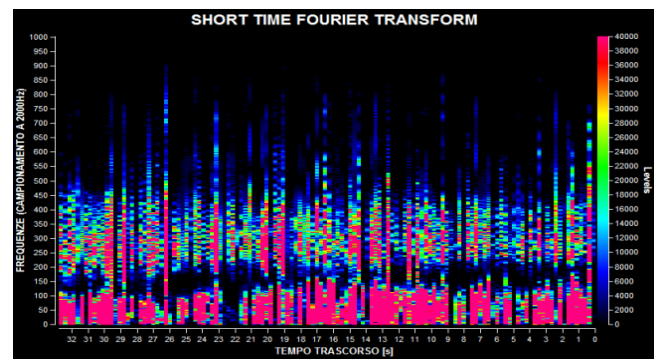

Fig. 7- Auscultation of the heart in aortic area with electronic stethoscope

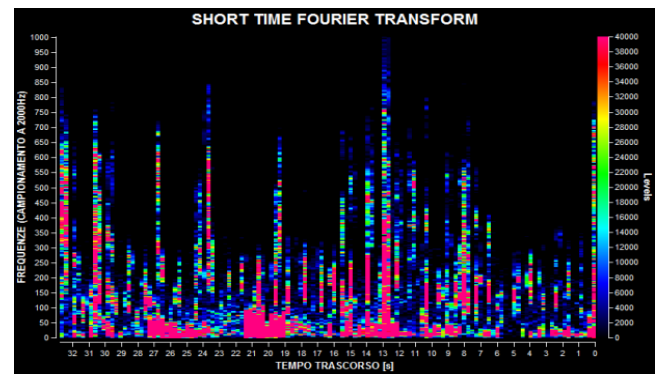

Fig. 8- Auscultation of the heart in aortic area with the new device

Figure 9 shows the sound spectrum of a heart sound concerning the tricuspid outbreak acquired and recorded by the reference electronic stethoscope and in Figure 10, the same sound was acquired by the new device.

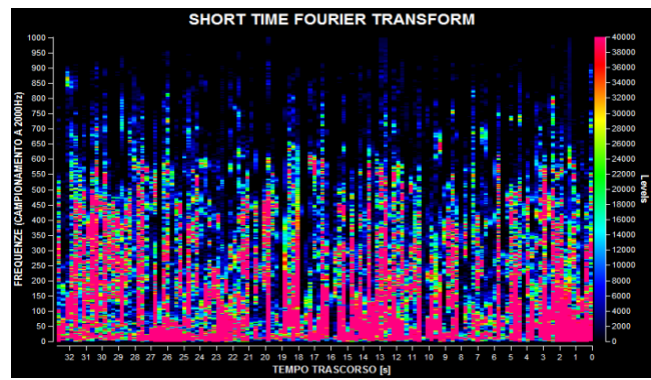

Fig. 9- Auscultation of the heart in tricuspid area with electronic stethoscope

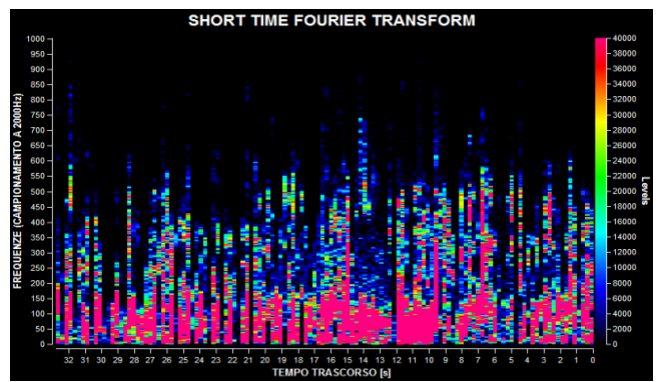

Fig. 10- Auscultation of the heart in tricuspid area with the new device

In all cases, the diagnosis obtained via the new device and by reference stethoscope is comparable.

Remote transmission test were also performed to assess the feasibility and accuracy of Web-based Heart and Lung sounds Auscultation (W) HLA in comparison to Traditional (T) HLA.

For this purpose have been studied 21 patients in a routine set- ting of the Cardiomyopathy Unit of Policlinico di Bari. Each patient was assessed by two expert cardiologists (Obs1-2) in two consecutive steps (W-HLA and T-HLA) using a cross-over study design. W-HLA was performed by using the internet tele-stethoscope. The high-quality audio signal was transmitted over the Internet by standard ADSL connection from the internet tele-stethoscope to a remote personal computer where the observer was able to hear and record $\mathrm{HLA}$ audio for W-HLA

A trained nurse positioned the stethoscope on topographical areas under webcam-assisted audiovisual guidance. T-HLA was performed by traditional binaural stethoscopes. Auscultatory findings were assessed by pre-classified values. Data were analyzed for concordance and tested by Fisher's exact test $(p<0.05)$ and kappa-test.

The results are summarized in figure 11 in terms of intraobserver concordance of W- vs T-HLA (No. of concordant findings, (\%)) for Obs1 and Obs2 for the overall findings, for heart sounds, and for pulmonary findings; and in terms of interobserver concordance of Obs1 vs Obs2 for the same findings.

\begin{tabular}{l|c|c|c|c|}
\multirow{2}{*}{ FINDINGS } & \multicolumn{2}{|c|}{ INTRA } & \multicolumn{2}{c|}{ INTER } \\
& WEB versus ACOUSTIC & \multicolumn{2}{c|}{ OBS 1 versus OBS 2 } \\
\hline \multirow{2}{*}{ Overall } & OBS 1 & OBS 2 & WEB & ACOUSTIC \\
& $217 / 231$ & $214 / 231$ & $224 / 231$ & $219 / 231$ \\
& $(93.9 \%)$ & $(92.7 \%)$ & $(96.8 \%)$ & $(94.8 \%)$ \\
\hline Heart & $81 / 84$ & $79 / 84$ & $79 / 84$ & $83 / 84$ \\
sounds & $(95.2 \%)$ & $(94.1 \%)$ & $(94.0 \%)$ & $(98.8 \%)$ \\
\hline Pulmonary & $60 / 63$ & $59 / 63$ & $59 / 63$ & $62 / 63$ \\
\hline $\begin{array}{l}\text { Systolic heart } \\
\text { murmur } \\
\text { chronology }\end{array}$ & $(95.2 \%)$ & $(93.6 \%)$ & $(93.6 \%)$ & $(98.4 \%)$ \\
\hline $\begin{array}{l}\text { Pulmonary } \\
\text { rales }\end{array}$ & $20 / 21(90.5 \%)$ & $18 / 21$ & $17 / 21$ & $19 / 21(90.5 \%)$ \\
& $\kappa=0.82$ & $(85.7 \%)$ & $(80.1 \%)$ & $\kappa=0.82$ \\
\hline
\end{tabular}

Fig. 11- Validation statistics for the internet tele-stethoscope

Therefore, heart and lung auscultation, as assessed by concordance analysis in our patient series, yielded high concordance of auscultatory findings for the traditional and web approach thanks to the new designed device. Intra- and interobserver concordance were not different for the two observers in the two settings. Thus, web heart and lung auscultation is a promising method for telemonitoring of patients affected by heart failure and the designed internet tele-stethoscope is reliable.

\section{Conclusions}

The designed device, able to objectify and to send biological sounds in real-time via web, seems to be of great interest both to improve the diagnostic potential of one of the most simple, fast, and completely devoid of drawbacks medical examination such auscultation, and for telemedicine applications that now seems a fixed course for reducing healthcare costs and improve quality of life of chronically ill patients through the implementation of treatment protocols in home care.

The device is also very useful for monitoring patients during therapy and to evaluate through accurate comparisons of auscultation reports the evolution of a disease. This type of monitoring, being objective, is to be shared with other doctors.

For the academic point of view, could be a great training tool. 


\section{References}

[1] Gross V., Dittmar A., Penzel T., Schuttler F., Von Wichert P. (2000) American Journal of Respiratory and Critical Care Medicine, 162, 905-909.

[2] Gavriely N., Nissan M., Rubin A.H., Cugell D.W. (1995) Thorax, 50, 1292-1300.

[3] Jingping X., Cheng J., Yanjun W. (1998) IEEE Transactions On Biomedical Engineering, 45(5), 660-663.

[4] Wodicka G.R., Stevens K.N., Golub H.L., Shannon D.C. (1990) IEEE Transactions On Biomedical Engineering, 37(12), 1130-1135.

[5] Hadjleontiadis L.J., Panas S.M. (1997) IEEE Transactions On Biomedical Engineering, 44(7), 642-648.

[6] Lu Y.S., Liu W.H., Quin G.X. (1988) The 10th Annual International Conference of the IEEE EMBS, 1, 175-176.

[7] Homs-Corbera W.A., Janè R., Fiz J., Morera J.A. (2000) The 22nd Annual International Conference of the IEEE EMBS, 1, 2977-2980.

[8] Paris D., Mastrorocostas A., Tolias Y.A., Theocharis J.B., Hadjilentiadis L.J., Panas S.M. (2000) IEEE Transactions On Biomedical Engineering, 47(9), 1165-1176.

[9] Earis E.J.E., Cheetam B.M.G. (2000) European Respiratory Review, 10(77), 586-590

[10]Giorgio A. (2011) Telemedicine: techniques and applications, In Tech Editor, ISBN 979-953-307-008-6. 